\title{
Misalignment Identification in Induction Motors Using Orbital Pattern Analysis
}

\author{
José Juan Carbajal-Hernández ${ }^{1, *}$, Luis Pastor Sánchez-Fernández ${ }^{1}$, \\ Victor Manuel Landassuri-Moreno ${ }^{2}$, and José de Jesús Medel-Juárez ${ }^{1}$ \\ ${ }^{1}$ Center of Computer Research - National Polytechnic Institute. Av. Juan de Dios Bátiz s/n, \\ Nueva. Industrial Vallejo, Gustavo A. Madero, México D.F., C.P. 07738, México \\ ${ }^{2}$ Mexico Valley University Center (CUUAEM-VM) - Autonomous University of the State \\ of Mexico. Boulevard Universitario, Predio San Javier, Atizapán de Zaragoza, \\ Estado de México, C.P. 54500, México \\ $\{j$ carbajalh, lsanchez, jjmedelj\}@cic.ipn.mx, \\ vmlandassurim@uaemex.mx
}

\begin{abstract}
Induction motors are the most common engine used worldwide. When they are summited to extensive working journals, e.g. in industry, faults may appear, generating a performance reduction on them. Several works have been focused on detecting early mechanical and electrical faults before damage appears in the motor. However, the main drawback of them is the complexity on the motor's signal mathematical processing. In this paper, a new methodology is proposed for detecting misalignment faults in induction motors. Through signal vibration and orbital analysis, misalignment faults are studied, generating characteristically patterns that are used for fault identification. Artificial Neural Networks are evolved with an evolutionary algorithm for misalignment pattern recognition, using two databases (training and recovering respectively). The results obtained, indicate a good performance of Artificial Neural Networks with low confusion rates, using experimental patterns obtained from real situations where motors present a certain level of misalignment.
\end{abstract}

Keywords: Orbital analysis, patterns recognition, neural networks evolution, motor fault, misalignment.

\section{Introduction}

Motor fault analysis is a common industrial practice where machinery is summited to extensive working journals. Induction motors are based on different electrical and mechanical components that can suffer some kind of wearing down with prolonged use [1]. This work has been oriented to electrical induction motors, since they are most used in industry worldwide. Historically, some works have been focused to detect some faults (most of them commonly identified) avoiding future problems and damages if correctly maintenance is early provided [1-3]. Induction motors can be mainly classified by size, power, number of phases, etc. However, misalignment in

* Corresponding author. 
rotor bars is a common fault in all kinds of motors that generates different levels of external vibrations [4].

Several techniques can be used in fault motor analysis such as support vector machine [5], Fourier spectrum [6], wavelet filtering [7], among others as [8-11]. However, the complexity of the mathematical processing in the motor signal is a drawback on them, where the implementation in real systems may be a difficult task. Thus, a new technic using orbital analysis is proposed, offering a practical and easy way to recognize misalignment faults in induction motors. Orbital analysis in motor faults has been used for modeling normal operation [12, 13], and they represent a basis of this research. Different motor misalignments levels present a particular characteristic vibration orbit, which can be used to determine when a motor presents a fault, before a serious damage appears in the machine. The main contribution of this work is the development of a new computational model for induction motors fault recognition, using artificial intelligence technics as Artificial Neural Networks (ANNs). They are evolved with an evolutionary algorithm called FS-EPNet to optimize the networks architectures for orbital analysis.

The rest of the paper is organized as follows: firstly, section 2 presents how electrical signals are measured and preprocessed using sensors, and how an orbit is created. Section 3 explains the representation of different misalignment faults in orbital patterns and the main characteristics over normal and bad orbits. In section 4 , ANNs and the FS-EPNet algorithm are shown for the recognition of orbital patterns. Thus, section 5 shows experimental results using two databases from real measured patterns: learning and recalling phases respectively. Finally, section 6 presents the conclusions reached about the advantages and disadvantages of the proposed model.

\section{$2 \quad$ Signal Acquisition and Preprocessing}

This section is aimed to present the procedure of obtaining a characteristic orbit, i.e. sampling, positioning and signal preprocessing to extract and separate it from a measured signal.

\section{Sampling}

Vibration is a common symptom derived from mechanical faults in induction motors. Such vibrations can be measured using a piezoelectric accelerometer sensor, which generates an electrical signal that is proportional to the acceleration vibration of a seismic mass [14]. As each motor have a different rotation speed, standards as [15] and [16] have established sampling frequency rates for motor measuring. According to them, this work used a sampling frequency of $50 \mathrm{kHz}$, being large enough to obtain a good quality signal, over tested induction motors.

\section{Positioning}

Orbital patterns are built using two signals that are plotted together. In order to obtain those signals, two piezoelectric accelerometers are placed orthogonally and radial to the motor chassis bearing (Fig. 1). 

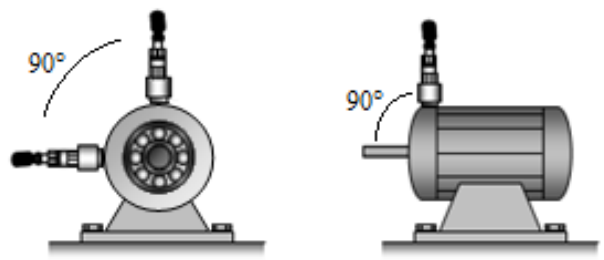

Fig. 1. Accelerometer placement at $90^{\circ}$ over the engine

\section{Signal Preprocessing}

Accelerometer signals are measured in acceleration units and must be converted to displacement units, using a double integration process as follows [14]:

$$
\begin{aligned}
& v(t)=\int_{0}^{t} a(t) d t+v_{0} \\
& d(t)=\int_{0}^{t} v(t) d t+d_{0}
\end{aligned}
$$

where $a(t)$ is acceleration, $v(t)$ is velocity, $d(t)$ is displacement, $v_{0}$ and $d_{0}$ are the initial velocity and displacement conditions respectively.

Vibration signals in displacement units are compounded by several harmonics; each of them can be related with the normal operation of the engine or with a motor fault. Undesirable harmonics can distort the shape of the orbit, changing notably the main characteristics of a fault shape. In this sense, those harmonics must be avoided in order to have a good quality orbit. A Butterworth passband filter was implemented for removing those spurious harmonics according to the following magnitude response [17]:

$$
|H(\omega)|^{2}=\frac{1}{1+\left(\frac{c-\cos \omega}{\Omega_{0} \sin \omega}\right)^{2 N}}
$$

where $\omega=2 \pi f / f_{s}, f_{s}$ is the sampling frequency, $\Omega_{0}=\tan \left(\omega_{0} / 2\right)$ and $c$ can be expressed as follows:

$$
c=\frac{\sin \left(\omega_{p a}+\omega_{p b}\right)}{\sin \omega_{p a}+\sin \omega_{p b}}
$$

where $\omega_{p a}=2 \pi f_{p a} / f_{s}, \omega_{p b}=2 \pi f_{p b} / f_{s}$ and $\left[f_{p a}, f_{p b}\right]$ is the passband.

An unfiltered orbit has an irregular form, making no possible fault discovery; however, a remarkable shape may be clearly seen in a filtered orbit (Fig. 2).

Each filtered signal generates continuous orbits with the same shape (Fig. 2); nevertheless, just one orbit is required in this work for analyzing its characteristics 

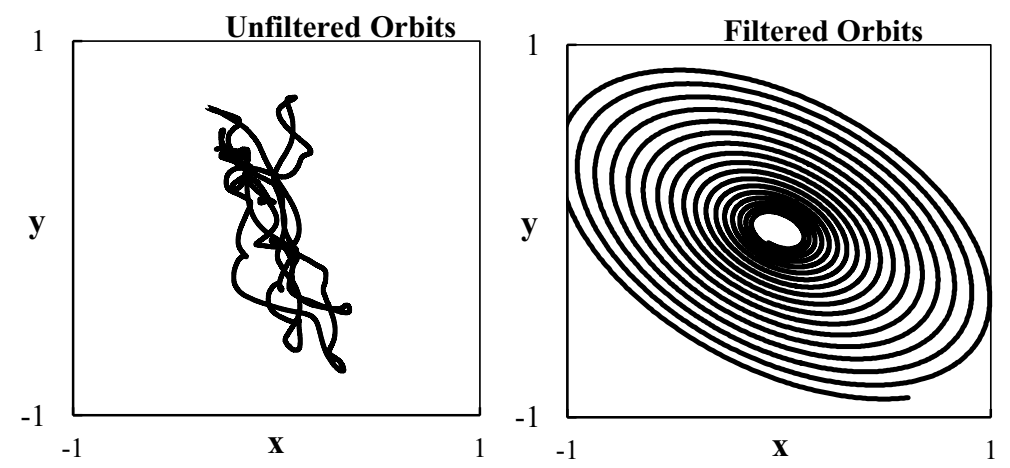

Fig. 2. Example of the orbital filtering using the Butterworth passband filter, which removes spurious harmonics allowing clear orbits

and detecting whether a misalignment is present. In this case, we search two points into a filtered signal (starting and ending), with a low distance defined by a tolerance. This tolerance was obtained computing the average of the distance of all points into the signal. There is not a rule for establishing the tolerance; however, this value was enough for obtaining good shape orbits. The distance criteria were obtained using the Euclidian equation as follows [17]:

$$
d=\sqrt{\left(x_{2}-x_{1}\right)^{2}+\left(y_{2}-y_{1}\right)^{2}}
$$

Where $d$ is the distance between points, and $(x, y)$ are the orbit points coordinates respectively. Finally, extracted orbits should be normalized due to differences in their size. Therefore, all orbits are resized in a $[-1,1]$ range according with the following equation:

$$
s_{1,2}(n)=\frac{d_{1,2}(n)}{\max \left\{\left|d_{1}(n)\right|,\left|d_{2}(n)\right|\right\}}, \quad \forall n=0,1,2, \ldots, N-1
$$

\section{Orbital Analysis}

There is a correspondence between orbit shapes and motor faults, i.e. when an induction motor is in good condition (no faults are present), the corresponding orbit is a circumference; on the other hand, when a misalignment fault is present in the motor, the orbit shape suffers a deformation in one part of the circumference. A misalignment may appear in different intensities: a slight misalignment almost deforms the circumference; by contrast, a strong misalignment deforms considerably the orbit shape, generating a kind of " 8 " in the circumference. Fig. 3 shows the motor's orbit shapes of different misalignment intensities. 

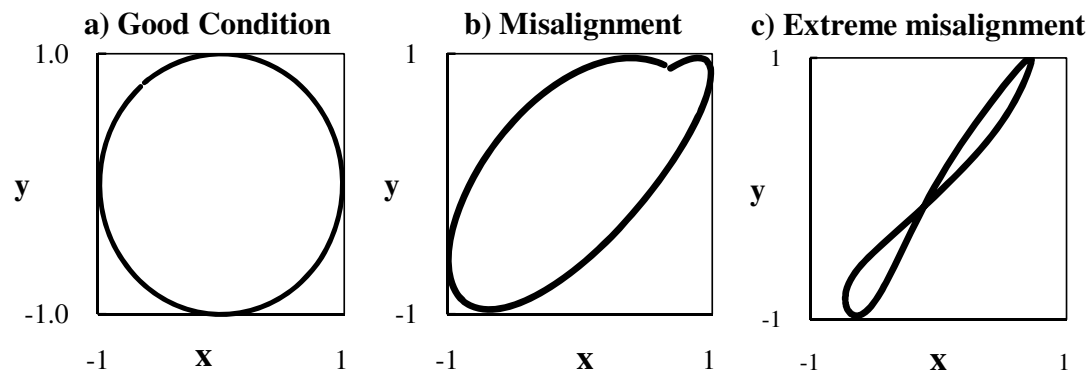

Fig. 3. Examples of orbit shapes: a) good motor conditions have perfect circumferences, b) a slight misalignment fault is present in the motor and c) an extreme misalignment have a shape of an "8" number.

\section{$4 \quad$ Pattern Recognition}

There are several technics for pattern recognition that can be used for orbits shape identification [1-11]. In this work, Artificial Neural Networks (ANN) are used as classifiers, because they have proved being a very effective learning model with high rates of effectiveness. However, the construction of an ANN is not an easy task; for this reason, evolutionary algorithms are used for building ANN architectures, establishing criteria for a better selection of the ANN's parameters. In this sense, the final ANN topology chosen by the evolutionary algorithm guarantees the best performance of the ANN. Also, connectivity reduction tests help to avoid computational burden with a high efficiency of the ANN. This process is known as Evolutionary Artificial Neural Networks (EANNs) or Neuroevolution.

\section{Artificial Neural Network}

Evolution of Artificial Neural Networks have been remarkably useful at optimizing networks' parameters during evolution [18-21], also local minima may be avoided than using traditional gradient-based search algorithms [18].

The Feature Selection EPNet algorithm (FS-EPNet) [19, 21] allows the ANNs' parameter evolution, including the input adaptation of the networks (Feature Selection Evolution). The FS-EPNet is a steady-state algorithm based in Lamarkian inheritance, where information learned by parents is passed to children; also, no crossover operator is used to avoid the permutation problems [18]. In this way, nine different mutations are used to carry out the evolution of individuals (ANNs): (1) hybrid training, composed of training with the Modified Back Propagation (MBP) algorithm and Simulated Annealing (SA); (2) node deletion; (3) connection deletion; (4) input deletion; (5) delay deletion; (6) connection addition; (7) node addition; (8) input addition; and (9) delay addition. Only one such mutation is performed on the selected individual in each generation. The hierarchical order of the mutations permit to maintain networks sizes to the minimum; however, if the problem cannot be solved more accurately, it will start to add nodes and connections, increasing the average 
networks sizes over the population. A detailed description of FS-EPNet algorithm may be seen in $[20,21]$.

\section{Pattern Building}

According with the orbital signal analysis, orbit shapes where used for creating motor fault patterns. However, resulting signal orbits are not practical to be used in a neural network due to they have different lengths. In order to have uniform patterns, all orbits signals were resampled for having 100 points of length, where each one is a bidimensional pattern $(x, y)$. A database of 386 patterns was created to be used in the learning phase of the ANN (from here, an inside test set is obtained to evolve the networks with the FS-EPNet algorithm). Orbit shapes of this database were measured from different induction motors, which had different misalignment levels: 275 regular misalignment patterns, 106 extreme misalignments patterns and 5 patterns of good condition motors.

\section{$5 \quad$ Experimental Results}

An experimental database was used for validating the performance of the proposed system as part of a recovering process. This database was built using different kind of motors and with different levels of misalignments. It is important to remark that this database was compounded by different motor measurements than those used in the database of the learning process. In this case, the size of the database was of 118 motor fault patterns as follows: 73 regular misalignment patterns, 35 extreme misalignments patterns and 10 patterns of good condition motors. From those patterns, the final test set was obtained, applied after the evolutionary process has finished. Preliminary experiments allow setting up some common parameters in this study: population size 30, generations of evolution 100 (stopping criteria), initial connection density $100 \%$ and $30 \%$. Initial learning rate 0.15 , minimum learning rate 0.01 , epochs for learning rate adaptation 5, number of mutated hidden nodes 1 , number of mutated connections 1-3. The inputs are fixed at 200, where the first half is for $x$-axis and the other half for $y$-axis. The hidden nodes are initialized between 2 and 10 nodes randomly. Partial training settle at 100 epochs, whereas 1000 epochs of further training at the end of the algorithm. 30 independent runs were performed to ensure statistical validity of the results. It was used two test sets to evaluate the performance of the algorithm, one inside of the evolutionary algorithm (100 partners from the available data to train) and one final test set (experimental database) to evaluate the final performance of the algorithm.

Figure 4 presents the Average Classification error (Fig. 4a), the average error in terms of the Normalize Root Mean Squared Error (NRMSE, Fig. 4b), the Average connections (Fig. 4c) and the Average hidden nodes (Fig. 4d) for the orbit motor fault recognition process over 100 generations of evolution with $100 \%$ and $30 \%$ of connectivity at network initialization. It can be seen in Fig. 4a, that initializing the networks with $100 \%$ of connectivity allows a perfect classification error in the test set inside the FS-EPNet algorithm, using the winner takes all method from the first 

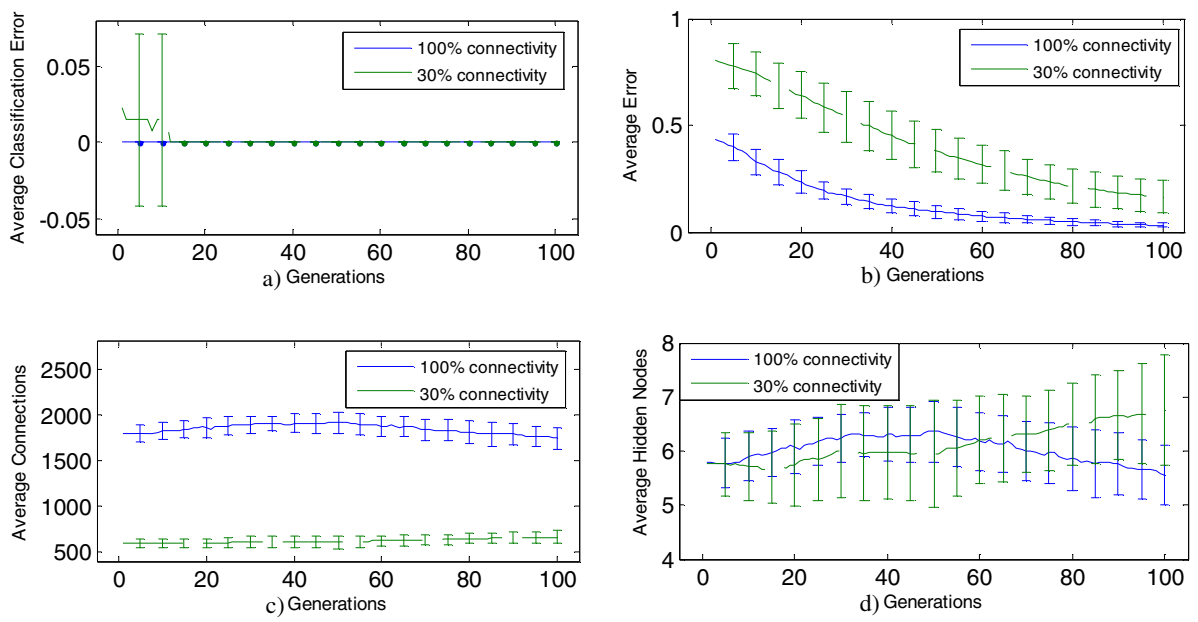

Fig. 4. Evolved parameters with the FS-EPNet over 100 generations of evolution for initial connectivity of $100 \%$ and $30 \%$ for the orbit motor fault recognition process: a) average classification error (winner takes all); b) average error in terms of the NRMSE; c) average connections and d) average hidden nodes.

generation. These results indicate that at the random initialization and initial partial training, the networks in the population can solve the problem without any effort; however, that is not maintained for the final test set (see final line in Table 1, for the final test set). On the other hand, a considerable reduction in the connectivity $(30 \%)$ produces average errors over 0.01 during the first 10 generations (Fig. 4a). Thereafter, the networks can solve the problem with the same accuracy, and with fewer numbers of connections (Fig. 4c). A similar behaviour is presented using the NRMSE over the test set inside the evolutionary algorithm, where both errors started to converge as the generation advance. Finally, as it can be seen in Fig. 4d, networks that are initialized with a reduce number of connections, started to increase the number of hidden nodes, as more resources continue to solve the problems accurately (also connections are slightly increased, Fig. 4c). It is clear that in both cases, 100 generations of evolutions is enough to achieve perfect classification errors on the test set inside the algorithm.

Table 1. Orbit motor fault recognition results with $100 \%$ and $30 \%$ of connectivity with the FS-EPNet

\begin{tabular}{lrrrrrrrr}
\hline \multicolumn{1}{c}{ Parameter } & \multicolumn{3}{c}{$100 \%$ Connectivity } & \multicolumn{3}{c}{ 30\% Connectivity } \\
\hline & Mean & Std Dev & Min & Max & Mean & Std Dev & Min & Max \\
\cline { 2 - 8 } Number of Inputs & 200 & 0 & 200 & 200 & 200 & 0 & 200 & 200 \\
Number of Hidden Nodes & 5.966 & 0.999 & 4 & 8 & 6.633 & 1.325 & 4 & 9 \\
Number of Connections & 1830.4 & 208.01 & 1419 & 2256 & 656.26 & 93.657 & 463 & 822 \\
Error Test Set EPNet & 0.034 & 0.01 & 0.018 & 0.061 & 0.168 & 0.084 & 0.023 & 0.333 \\
Classification Error inside & 0 & 0 & 0 & 0 & 0 & 0 & 0 & 0 \\
Error Final Test Set & $\mathbf{4 . 0 5 4}$ & $\mathbf{0 . 2 0 7}$ & $\mathbf{3 . 7 5 3}$ & $\mathbf{4 . 4 0 5}$ & $\mathbf{4 . 2 8 1}$ & $\mathbf{0 . 4 2 7}$ & $\mathbf{3 . 2 9 7}$ & $\mathbf{5 . 0 5 4}$ \\
Classification Error Final Test Set & $\mathbf{6 . 3 7 1}$ & $\mathbf{0 . 9 8 7}$ & $\mathbf{5 . 1 2 8}$ & $\mathbf{8 . 1 5 8}$ & $\mathbf{6 . 7 3 6}$ & $\mathbf{1 . 0 2 8}$ & $\mathbf{4 . 8 9 5}$ & $\mathbf{8 . 6 2 4}$ \\
\hline
\end{tabular}


Table 1 presents the results of evolving ANNs with both values of connectivity. There is appreciated that classification errors tested during the evolution of the networks is perfect, as commented before (over the classification error inside the evolutionary algorithm); nevertheless, that is not maintained for the final test set, last line of Table 1.

\section{Discussion and Conclusions}

In this work, the use of orbital analysis and evolved Artificial Neural Networks (ANNs) for fault recognition in induction motor were proposed. Although several methodologies for detecting mechanical faults in induction motor have been developed, the proposed model represents a feasible and alternative way for motor misalignment fault detection. One disadvantage of this model is the number of preprocessing steps implemented before the ANN classification step. However, a misalignment was clearly shown to distort considerably an orbit, having a characteristic shape, which can be perfectly identified by classifiers as ANNs (designed with the FS-EPNet algorithm). On the other hand, the evolution of Artificial Neural Network provides a good topology optimization, avoiding computational burden for recovering phase, and giving an accurate assessment in the classification of misalignment orbits. It may be worth to say that this paper provides a preliminary study of misalignment identification in induction motors using orbital pattern analysis, and future works is needed, e.g. including additional patterns from different mechanical faults in order to expand the capacities of the system, or use lower connectivity values to initialize ANNs (before evolution starts) to generate smaller architectures. Anyhow, this model can be used as an important tool for preliminaries motor analysis, when the good functioning of the machine is essential in critical time production industry.

\section{References}

1. Chow, M.: Methodologies of using neural network and fuzzy logic technologies for motor incipient fault detection. World Scientific, Singapore (1997)

2. Wang, J., Gao, R., Yan, R.: Broken-rotor-bar diagnosis for induction motors. Journal of Physics 305, 1-10 (2011)

3. Filippetti, F., Franceschini, G., Tassoni, C.: Neural networks aided on-line diagnostics of induction motor rotor faults. IEEE Transactions on Industry Applications 31(4), 892-899 (1995)

4. Lee, Y., Lee, C.: Modelling and analysis of misaligned rotor-ball bearing systems. Journal of Sound and Vibration 224, 17-32 (1999)

5. Matić, D., Kulić, F.: SVM broken bar detection based on analysis of phase current. In: 5th International Power Electronics and Motion Control Conference and Exposition, vol. (6397456), pp. LS4c.21-LS4c.24 (2012)

6. Climente, V., Antonino, J., Riera, M., Puche, R., Escobar, L.: Application of the WignerVille distribution for the detection of rotor asymmetries and eccentricity through highorder harmonics. Electric Power Systems Research 91, 28-36 (2012) 
7. Kechida, R., Menacer, A., Talhaoui, H.: Approach signal for rotor fault detection in induction motors. Journal of Failure Analysis and Prevention 13(3), 346-352 (2013)

8. Füssel, D., Ballé, P.: Combining neuro-fuzzy and machine learning for fault diagnosis of a D.C. motor. In: Proceedings of the American Control Conference, pp. 37-41 (1997)

9. Gaylard, A., Meyer, A., Landy, C.: Acoustic evaluation of faults in electrical machines. In: Electrical Machines and Drives, Conference Publication; 412, pp. 147-150 (1995)

10. Liu, D., Zhao, Y., Yang, B., Sun, J.: A new motor fault detection method using multiple window S-method time-frequency analysis. In: International Conference on Systems and Informatics, pp. 2563-2566 (2012)

11. Kim, K., Parlos, A.: Induction motor fault diagnosis based on neuropredictors and wavelet signal processing. IEEE/ASME Transactions on Mechatronics 7(2), 201-219 (2002)

12. Ha, K., Hong, J., Kim, G., Chang, K., Lee, J.: Orbital analysis of rotor due to electromagnetic force for switched reluctance motor. IEEE Transactions on Magnetics 36(4), 1407-1411 (2000)

13. Dongfeng, S., Lianfsheng, O., Ming, B.: Instantaneous purified orbit: A new tool for analysis of nonstationary vibration of rotor system. International Journal of Rotating Machinery 7(2), 105-115 (2001)

14. Han, S.: Retrieving the time history of displacement from measured acceleration signal. Journal of Mechanical Science and Technology 17(2), 197-206 (2003)

15. ISO 10816. Mechanical vibration: evaluation of machine vibration by measurements on non-rotating parts (1995)

16. VDI 2056. Standards of evaluation for mechanical vibrations of machines, Germany (1964)

17. Proakis, J., Manolakis, D.: Tratamiento digital de señales. Pearson Education, vol. 1(4a). Ed. España (2007)

18. Yao, X., Liu, Y.: A new evolutionary system for evolving artificial neural networks. IEEE Transactions on Neural Networks 8(3), 694-713 (1997)

19. Yao, X.: Evolving artificial neural networks. Proceedings of the IEEE 87(9), 1423-1447 (1999)

20. Bullinaria, J.: Evolving neural networks: Is it really worth the effort? In: Proceedings of the European Symposium on Artificial Neural Networks, pp. 267-272. d-side, Evere (2005) 